\title{
Polylactic Acid (PLA): Improve It, Use It, and Dump It Faster
}

Narongrit Sombatsompop, ${ }^{\mathrm{a}, *}$ Panupong Srimalanon, ${ }^{\mathrm{a}}$ Teerasak Markpin, ${ }^{\mathrm{a}}$ and Benjaphorn Prapagdee ${ }^{b}$

Today, many people enjoy an easy lifestyle. However, this comfort has come with a price because of plastic that is thrown away after a single use. As such, governments around the world have pushed for biodegradable plastics to be produced, especially for food packaging, and these can be easily seen in supermarkets, for example. Using plastic for only one time has resulted in environmental pollution. To solve this problem, polylactic acid (PLA) has been introduced as an alternative bio-based plastic to replace artificial petroleum-based plastics. PLA comes from renewable resources and is biodegradable under certain conditions. Furthermore, the development of the properties of PLA could solve problems related to its weakness in packaging applications. This editorial proposes expansion of the property attributes of PLA to include hygienic character, through the addition of antibacterial agents. This can be done by introducing two alternative approaches for waste management: PLA recycling and degradation. However, some key research is still needed to improve the properties and waste management of PLA relative to the effectiveness of its reprocessing and acceleration of its (bio)degradation.

Keywords: Polylactic acid; PLA waste management; Biodegradation; Packaging

Contact information: a: Polymer PROcessing and Flow (P-PROF) Research Group, Division of Materials Technology, School of Energy, Environment and Materials, King Mongkut's University of Technology Thonburi (KMUTT), Thungkru, Bangkok 10140, Thailand; b: Laboratory of Environmental Biotechnology, Faculty of Environment and Resource Studies, Mahidol University, Salaya, Nakhon Pathom 73170, Thailand; * Correspondence author: narongrit.som@ kmutt.ac.th

\section{PLA as an Alternative Bio-based Plastic and Property Improvement}

A wide variety of synthetic or petroleum-based plastics have been produced and used worldwide to support the demand of plastic consumption for human activities, mainly for packaging applications. Most packaging products are synthetic or petroleum-based plastics, e.g., polyethylene terephthalate, polyethylene, polypropylene, and polystyrene. In 2020, the demand for plastic packaging for home-delivered groceries has significantly increased during the COVID-19 lockdowns. Synthetic petroleum-based plastics are resistant against microbial degradation, resulting in persisting in the environment after disposal. Therefore, accumulation of petroleum-based plastic waste has been recognized as a critical environmental issue. The problem can also be more significant when the size of the plastics turns into microplastics. Microplastics can be potentially taken up and ingested by a number of edible marine animals, resulting in entrance into the food chain.

To minimise the quantities of the synthetic or petroleum-based waste, use of biodegradable plastics that can easily decompose after their disposal in the environment has received much interest and is being emphasized. Polylactic acid (PLA), a bio-based plastic, is one of the most common biodegradable plastics for packaging applications. 
Approximately 293,000 tonnes of PLA were produced in 2019, and this amount will continue to increase (European Bioplastics 2019). The results from a recent survey showed that the food, fruit, and vegetable packaging applications would still be dominant for PLA, and the growth in the packaging industry would be expected to increase significantly in developing regions such as the Asia-Pacific, South America, and the Middle East. The increase may result from promotional policies by the Food and Drug Administration (FDA) and related organisations for the usage of biodegradable plastics in these regions. PLA is produced from condensation polymerisation of lactic acid monomers, which are generated from natural and renewable resources, e.g., maize, tapioca, and sugar cane by a bacterial fermentation process. The material can be decomposed into carbon dioxide and water under aerobic conditions. After use, PLA waste management plays an important role through the approaches of recycling and biodegradation. A most recent review by Hubbe et al. (2021) addressed the formulation of bioplastic composites, focusing on PLA and poly(hydroxybutyrate) (PHB), for biodegradability, recycling, and performance.

Although PLA is biodegradable, non-toxic, with high tensile strength and high modulus properties, it is brittle with low impact strength, low elongation at break, and has low heat resistance. These drawbacks cause great restriction relative to its potential uses for packaging applications. In addition, based on the literature, PLA's properties have been improved by several approaches, which include additions of chain extenders and chemical compatibilisers or blending with other flexible biodegradable polymers. For example, it was reported that the addition of polybutylene succinate (PBS) at just $20 \mathrm{wt} \%$ could change the fracture behaviour of PLA from being brittle to ductile. The elongation at break and tensile toughness of the PBS/PLA blends was increased by adding a small amount of dicumyl peroxide (DCP).

\section{Hygienic PLA}

Hygienic PLA has been developed by the addition of antibacterial agents, not only in neat PLA, but also in natural fibre/PLA composites. Triclosan and hydroxypropyl-3piperazinyl-quinoline carboxylic acid methacrylate (HPQM) have been considered for such applications. Triclosan was incorporated in the PLA and wood/PLA composites. The resulting antibacterial performance of wood/PLA against the growth of Escherichia coli (E. coli) was better than that of neat PLA. This suggested that the presence of wood particles promoted antibacterial performance, and the wood particles acted as an 'antibacterial promoter' (Prapruddivongs and Sombatsompop 2012). The antibacterial agent HPQM, which is claimed to be environmentally friendly, was allowed for use in the food industry. The antibacterial activity of PLA and its blend with PBS was improved at the optimum concentrations of 1500 to 2000 ppm of HPQM.

\section{PLA Waste Management}

Owing to the use of enormous quantities of single-use and short shelf life PLA packaging products, the waste management issue for PLA disposal is a critical concern, and this issue is still open for discussion among scientists. Two alternative approaches of PLA waste management are proposed here, comprising PLA recycling and degradation. Firstly, PLA recycling is a common and feasible way, and this refers to the reprocessing of the PLA scraps from discarded PLA products in order to reduce the accumulation of the PLA waste. Based on the most recent literature, the number of feasible reprocessing cycles has been found to depend on the compositions of the PLA products. 
Secondly, the PLA waste could be degraded under soil burial or compost conditions, but the PLA degradation was relatively slow in soil at a mesophilic temperature ( 30 to $35^{\circ} \mathrm{C}$ ). The study by Pattanasuttichonlakul et al. (2018) stated that commercial PLA packaging products lost only 5 to $10 \%$ of their weight during biodegradation at an ambient condition for 60 days. The mechanical processes by a shredding method and UV radiation exposure of used PLA packaging could help reduce the molecular weights of the PLA, and this was found to promote the PLA degradation. In order to accelerate the PLA biodegradation, microbial bioaugmentation and nutrient biostimulation were applied. The potent PLA-degrading bacteria as bacterial inoculum for bioaugmentation were isolated. The authors found that Pseudonocardia sp. RM423 (Apinya et al. 2015) and Pseudomonas geniculate WS3 (Bubpachat et al. 2018) enhanced the PLA film biodegradation under submerged conditions. Soil inoculation with a specific PLA-degrading bacterium on accelerating the PLA biodegradation under soil burial was described in the authors' previous studies, suggesting an effective approach for PLA waste treatment (Apinya et al. 2015; Pattanasuttichonlakul et al. 2018).

To stimulate PLA biodegradation, an appropriate nitrogen source was applied to enhance the bacterial growth and the production of PLA-degrading enzymes from PLAdegrading bacteria. Gelatin, as a proteinous substance, had a positive stimulating effect on PLA biodegradation (Apinya et al. 2015). This acted as an enzymatic inducer by stimulating the production of protease and PLA-degrading enzymes by $P$. geniculate WS3 (Bubpachat et al. 2018). In addition, the study of Pattanasuttichonlakul et al. (2018) attempted to use wastewater sludge from a wide range of sources, such as from coconut milk, dairy, and rice vermicelli factories, to stimulate the PLA biodegradation under soil burial. They found that a complete degradation of PLA packaging appeared after 15 days of burial in the soil supplemented by a high nitrogen content of dairy waste water sludge. A combined use of both the biostimulation by the addition of soytone as an organic nitrogen and the bioaugmentation with a specific PLA-degrading bacterium could successfully accelerate the PLA biodegradation.

\section{Challenges and Prospective Research}

Packaging applications, especially for food and fruits, have been the major share for PLA usage due to the promotion regulations of the usage of biodegradable plastics across the globe. PLA also has an increasing market share in automotive, medical, and electronics applications and will show its role in 3D printing applications in the near future. If this is the case, further improvements of PLA properties would be potential research topics for scientists and technologists. Interestingly, the Scopus database has indicated that China, the United States, and India are the top three countries that have been publishing over 5000, 2000, and 1000 research papers, respectively on PLA during the past five years (2016 to 2020). Research topics on PLA under current investigation have still focused on the improvements of its mechanical properties, especially for sheet and film applications, and the biodegradability from abundant PLA products.

In relation to this editorial, the biodegradation of hygienic PLA products has been found to be retarded due to the microbial inhibitory effects of a residual antimicrobial agent in PLA during its service life. Therefore, the techniques or processes to overcome the effects of the remaining antibacterial agents in discarded PLA products must be further conducted before continuing through the disposal process. As a result of the potential of enzyme technology, the upscaled production of PLA-degrading enzymes and their 
application on stimulating the degradation of PLA packaging waste should be further investigated. Due to the limited information of the optimum conditions for accelerating the PLA degradation in the real environment, further studies on a pilot scale of the applications of the optimal nitrogen sources and PLA-degrading bacterial consortium (pure or mixed cultures) for PLA waste degradation in soil and/or compost should be initiated. Based on the success of accelerating the PLA biodegradation by microbial bioaugmentation, the potential effects of microbial species on PLA biodegradation need to be explored. Preparation of a "ready-to-use bacterial inoculum" for the field applications should also be developed. Therefore, the establishment of an effective innovative approach of PLA waste recycling and degradation could lead to more sustainable development of PLA waste management.

\section{References Cited}

Apinya, T., Sombatsompop, N., and Prapagdee, B. (2015). "Selection of a Pseudonocardia sp. RM423 that accelerates the biodegradation of poly(lactic) acid in submerged cultures and in soil microcosms," International Biodeterioration \& Biodegradation 99, 23-30. DOI: 10.1016/j.ibiod.2015.01.001

Bubpachat, T., Sombatsompop, N., and Prapagdee, B. (2018). "Isolation of two degrading bacteria and their roles on production of degrading enzymes and PLA biodegradability at mesophilic conditions," Polymer Degradation and Stability 152, 75-85. DOI: 10.1016/j.polymdegradstab.2018.03.023

European Bioplastics. (2019). Bioplastics Market Data 2019 (online) [Accessed date: $1^{\text {st }}$ November 2020], Available from: https://www.european-bioplastics.org/market/

Hubbe, M. A., Lavoine, N., Lucia, L. A., and Dou, C. (2021) "Formulating bioplastic composites for biodegradability, recycling, and performance: A review," BioResources 16(1), 2021-2083.

Pattanasuttichonlakul, W., Sombatsompop, N., and Prapagdee, B. (2018). “Accelerating biodegradation of PLA using microbial consortium from dairy wastewater sludge combined with PLA-degrading bacterium," International Biodeterioration \& Biodegradation 132, 74-83. DOI: 10.1016/j.ibiod.2018.05.014

Prapruddivongs, C., and Sombatsompop, N. (2012). "Roles and evidence of wood flour as an antibacterial promoter for triclosan-filled poly(lactic acid)," Composites: Part B: Engineering 43, 2730-2737. DOI: 10.1016/j.compositesb.2012.04.032 